\title{
$\left.{ }_{\text {SCIENCE }}^{\text {MATRIX }}\right\}$ Mascot Search Results
}

\section{Protein View}

Match to: OMPA_SALTY Score: 138 Expect: 2e-010

Outer membrane protein A OS=Salmonella typhimurium (strain LT2 / SGSC1412 / ATCC 700720) GN=ompA PE=3 SV=2

Nominal mass $\left(\mathrm{M}_{\mathrm{r}}\right)$ : $\mathbf{3 7 4 9 2}$; Calculated $\mathrm{pI}$ value: $\mathbf{5 . 6 0}$

NCBI BLAST search of OMPA SALTY against $\mathrm{nr}$

Unformatted sequence string for pasting into other applications

Taxonomy: Salmonella enterica subsp. enterica serovar Typhimurium str. LT2

Variable modifications: Carbamidomethyl (C), Oxidation (M)

Cleavage by Trypsin: cuts C-term side of $\mathrm{KR}$ unless next residue is $\mathrm{P}$

Number of mass values searched: $\mathbf{4 5}$

Number of mass values matched: 16

Sequence Coverage: 46\%

Matched peptides shown in Bold Red

1 MKKTAIAIAV ALAGFATVAQ AAPKDNTWYA GAKLGWSQYH DTGFIHNDGP

51 THENQLGAGA FGGYQVNPYV GFEMGYDWLG RMPYKGDNIN GAYKAQGVQI

101 TAKLGYPITD DLDVYTRLGG MVWRADTKSN VPGGPSTKDH DTGVSPVFAG

151 GIEYAITPEI ATRLEYQWTN NIGDANTIGT RPDNGLLSVG VSYRFGQQEA

201 APVVAPAPAP APEVQTKHFT LKSDVLFNFN KSTLKPEGQQ ALDQLYSQLS

251 NLDPKDGSVV VLGFTDRIGS DAYNQGLSEK RAQSVVDYLI SKGIPSDKIS

301 ARGMGESNPV TGNTCDNVKP RAALIDCLAP DRRVEIEVKG VKDVVTQPOA

351

Show predicted peptides also

Sort Peptides By $\odot$ Residue Number $\bigcirc$ Increasing Mass $\bigcirc$ Decreasing Mass

$\begin{array}{rrrr}\text { Start - End } & \text { Observed } & \text { Mr (expt) } & \text { Mr (calc) } \\ 25-33 & 1025.4610 & 1024.4537 & 1024.4614 \\ 104-117 & 1640.7993 & 1639.7920 & 1639.8094 \\ 118-124 & 818.4228 & 817.4155 & 817.4269 \\ 118-124 & 834.4288 & 833.4215 & 833.4218 \\ 139-163 & 2616.5466 & 2615.5394 & 2615.2868 \\ 195-217 & 2303.2975 & 2302.2902 & 2302.1957 \\ 223-231 & 1083.5485 & 1082.5413 & 1082.5397 \\ 256-267 & 1264.6321 & 1263.6248 & 1263.6460 \\ 268-280 & 1381.6573 & 1380.6500 & 1380.6521 \\ 268-281 & 1537.7366 & 1536.7293 & 1536.7532 \\ 281-292 & 1378.7443 & 1377.7371 & 1377.7616 \\ 303-321 & 2032.9644 & 2031.9572 & 2031.9102 \\ 322-332 & 1157.5886 & 1156.5813 & 1156.5910 \\ 322-332 & 1214.6054 & 1213.5982 & 1213.6125 \\ 322-333 & 1370.6942 & 1369.6869 & 1369.7136 \\ 333-339 & 872.4874 & 871.4801 & 871.5127\end{array}$

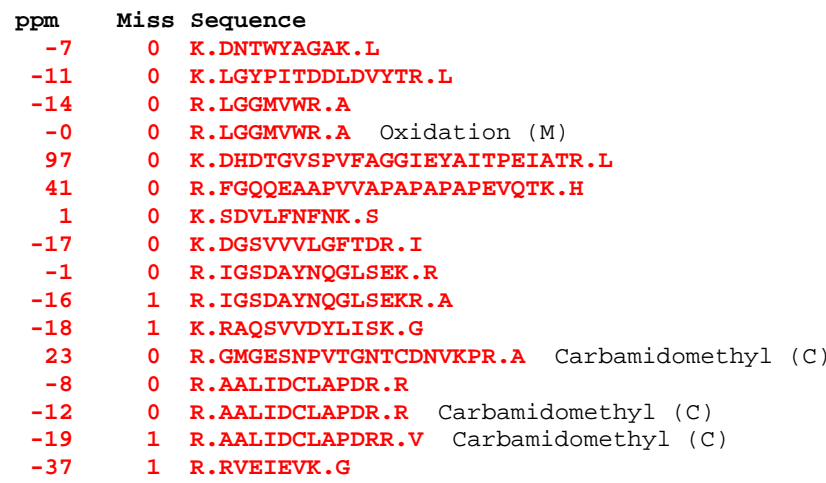

No match to: $608.3076,643.9765,662.2539,832.2619,833.0444,855.0124,870.9773,973.5256,1060.0080,1065.5099,1193.597 !$

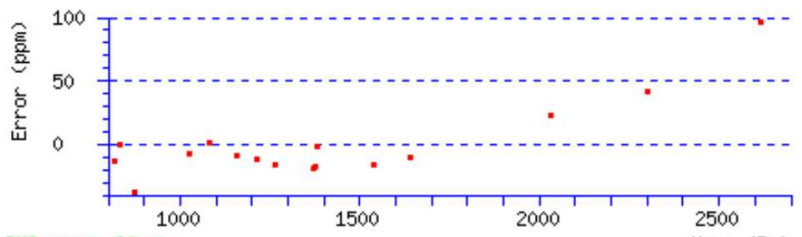

Mass (Da)

OMPA SALTY

P02936;

Reviewed;

350 AA.

21-JUL-1986, integrated into UniProtKB/Swiss-Prot.

23-JAN-2002, sequence version 2 .

16-MAY-2012, entry version 100 .

RecName: Full=Outer membrane protein $A_{i}$

AltName: Full=Outer membrane major heat-modifiable protein;

Alt Name: Full=outer membrane protein $33 \mathrm{~K}$

Flags: Precursor:

Name=ompA; OrderedLocusNames=STM1070;

Salmonella typhimurium (strain LT2 / SGSC1412 / ATCC 700720).

Bacteria; Proteobacteria; Gammaproteobacteria; Enterobacteriales;

Enterobacteriaceae; Salmonella;

Salmonella enterica subsp. enterica serovar Typhimurium.

NCBI_TaxID $=99287$.

[1]

NUCLEOTIDE SEQUENCE [GENOMIC DNA].

MEDLINE $=83287368 ;$ PubMed $=6349993$

DOI $=10.1111 / j .1432-1033.1983 . t b 07594 \cdot x ;$

Freudl R., Cole S.T.;

"Cloning and molecular characterization of the ompA gene from

Salmonella typhimurium."

Eur. J. Biochem. 134:497-502(1983).

[2]

NUCLEOTIDE SEQUENCE [LARGE SCALE GENOMIC DNA].

STRAIN=LT2 / SGSC1412 / ATCC 700720 ; 


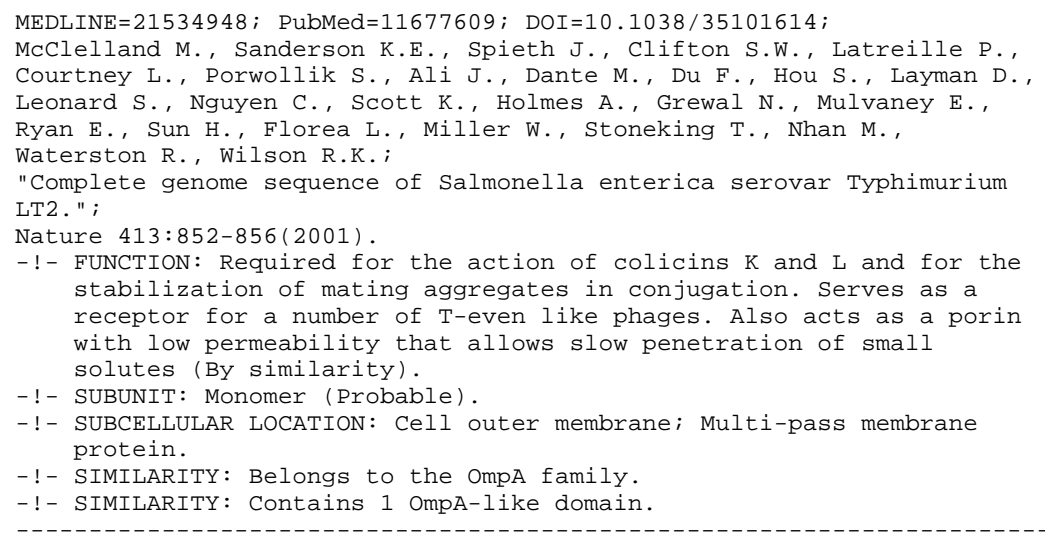

Copyrighted by the UniProt Consortium, see http://www uniprot.org/terms Distributed under the Creative Commons Attribution-NoDerivs License

EMBL; X02006; CAA26037.1; -; Genomic_DNA

EMBL; AE006468; AAL20003.1; -; Genomic_DNA.

PIR; A03436; MMEBAT.

RefSeq; NP_460044.1; NC_003197.1.

ProteinModelPortal; P02936; -

SMR; P02936; 22-196.

PRIDE; P02936; - .

GeneID; 1252588 ; - .

GenomeReviews; AE006468_GR; STM1070.

KEGG; stm:STM1070; - .

PATRIC; 32380577; VBISalEnt20916_1135.

eggNOG; COG2885; - .

HOGENOM; HOGO00274199; -

KO; K03286; - .

OMA; DDNEAOK; -

ProtClustDB; PRK10808; -

BioCyc; STYP99287:STM1070-MONOMER; -

GO; GO:0009279; C:cell outer membrane; IEA:UniProtKB-SubCell.

GO; GO:0005886; C:plasma membrane; IEA:UniProtKB-KW.

GO; GO:0046930; C:pore complex; IEA:UniProtKB-KW.

GO; GO:0015288; F:porin activity; IEA:UniProtKB-KW.

GO; GO:0005198; F:structural molecule activity; IEA:InterPro.

$\mathrm{GO}$; GO:0000746; P:conjugation; IEA:UniProtKB-KW.

GO; GO:0006811; P:ion transport; IEA:UniProtKB-KW.

Gene3D; G3DSA:2.40.160.20; OMP_b-brl; 1 .

Gene3D; G3DSA:3.30.1330.60; OmpA/MotB_C; 1 .

InterPro; IPR011250; OMP_b-brl.

InterPro; IPR006664; OMP bac.

InterPro; IPR002368; OmpA.

InterPro; IPR006690; OMPA-like_CS.

InterPro; IPR000498; OmpA-like_TM_dom.

InterPro; IPR006665; OmpA/MotB_C.

Pfam; PF00691; OmpA; 1 .

Pfam; PF01389; OmpA membrane; 1.

PRINTS; PR01021; OMPADOMAIN.

PRINTS; PR01022; OUTRMMBRANEA.

SUPFAM; SSF103088; OmPA/MOtB_C; 1.

PROSITE; PS01068; OMPA_1; 1 .

PROSITE; PS51123; OMPA_2; 1 .

3: Inferred from homology;

Cell membrane; Cell outer membrane; Complete proteome; Conjugation;

Disulfide bond; Ion transport; Membrane; Porin; Reference proteome;

Repeat; Signal; Transmembrane; Transmembrane beta strand; Transport.

SIGNAL $\quad 1 \quad 21 \quad$ By similarity.

CHAIN $22 \quad 350 \quad$ Outer membrane protein A.

/FTId=PRO_0000020099.

TRANSMEM $27 \quad 40 \quad$ Beta stranded; (Potential).

TRANSMEM $\quad 55 \quad 67 \quad$ Beta stranded; (Potential).

TRANSMEM $\quad 70 \quad 85 \quad$ Beta stranded; (Potential).

TRANSMEM $\quad 97 \quad 107 \quad$ Beta stranded; (Potential).

TRANSMEM $111 \quad 126 \quad$ Beta stranded; (Potential).

TRANSMEM $146 \quad 157 \quad$ Beta stranded; (Potential).

TRANSMEM $163 \quad 179 \quad$ Beta stranded; (Potential).

TRANSMEM $185 \quad 196 \quad$ Beta stranded; (Potential).

REPEAT $205 \quad 206 \quad 1$.

REPEAT $207 \quad 208 \quad 2$.

REPEAT $209 \quad 210 \quad 3$

$\begin{array}{llll}\text { REPEAT } & 211 & 212 & 4\end{array}$

DOMAIN $214 \quad 342 \quad$ OmpA-like.

REGION $205 \quad 212 \quad 4 \times 2$ AA tandem repeats of A-P.

DISULFID $315 \quad 327 \quad$ By similarity.

CONFLICT $114 \quad 114 \quad \mathrm{~V} \rightarrow \mathrm{F}$ (in Ref. 1; CAA26037).

CONFLICT $247 \quad 247 \quad S \rightarrow I$ (in Ref. 1; CAA26037).

SEQUENCE $350 \mathrm{AA}$; $37515 \mathrm{MW}$; B4AC52C8C5DF54FE CRC64;

MKKTAIAIAV ALAGFATVAQ AAPKDNTWYA GAKLGWSQYH DTGFIHNDGP THENQLGAGA FGGYQVNPYV GFEMGYDWLG RMPYKGDNIN GAYKAQGVQL TAKLGYPITD DLDVYTRLGG MVWRADTKSN VPGGPSTKDH DTGVSPVFAG GIEYAITPEI ATRLEYQWTN NIGDANTIGT RPDNGLLSVG VSYRFGQQEA APVVAPAPAP APEVQTKHFT LKSDVLFNFN KSTLKPEGQQ ALDOLYSOLS NLDPKDGSVV VLGFTDRIGS DAYNOGLSEK RAOSVVDYLI SKGIPSDKIS ARGMGESNPV TGNTCDNVKP RAALIDCLAP DRRVEIEVKG VKDVVTQPQA 